Original Research Article

\title{
A prospective observational pharmacovigilance study of adverse drug reaction monitoring in patients of MDR - TB at tertiary care hospital
}

\author{
Mirza Shiraz Baig1, Monali Raghunath Kale ${ }^{1 *}$, Avinash Lamb²
}

\begin{abstract}
${ }^{1}$ Department of Pharmacology,
${ }^{2}$ Department of Chest \& TB,

Government Medical College

Aurangabad, Maharashtra, India
\end{abstract}

Received: 27 February 2018

Revised: 18 April 2018

Accepted: 25 April 2018

\section{*Correspondence to:}

Dr. Monali Raghunath Kale,

Email: drmonalikale@

gmail.com

Copyright: (C) the author(s), publisher and licensee Medip Academy. This is an openaccess article distributed under the terms of the Creative Commons Attribution NonCommercial License, which permits unrestricted noncommercial use, distribution, and reproduction in any medium, provided the original work is properly cited.

\begin{abstract}
Background: The emergence of drug resistant mycobacteria has become a significant public health problem world over creating an obstacle to effective TB control. ADRs are common in patients of MDR-TB on DOTs-Plus drug regimen. Present study was carried out in tertiary care hospital. Identification of types and frequency of adverse drug reactions in Intensive and continuation phase of MDRTB Patients.

Methods: It was a prospective observational study conducted in Department of TB- Chest and Medicine, Govt. Medical College, Aurangabad, Maharashtra, India. All the MDR-TB patients admitted at the directly observed treatment, short course plus (DOTS plus) Center at Medical College Hospital were enrolled and were monitored for ADRs. The causality and severity of the reactions were determined using Naranjo algorithm and Hartwig questionnaire, respectively.

Results: A total of 121 tuberculosis patients of MDR-TB on DOTS therapy were enrolled for the study. Out of 121 patients, 13 were dropouts, 6 died, 7 defaulted so 108 patients assessed for ADRs, 48 patients developed $61(56.48 \%)$ adverse drug reactions. The higher numbers of ADRs were observed in age group 31$40 \mathrm{yrs}$ followed by 21-30yrs which were more common in men. Majority of adverse drug reactions were Gastrointestinal (GI) problems 32 (52.45\%), followed by Ototoxicity 7 (11.48\%) and Psychiatric Manifestations 6 (9.84\%) and skin problems $3(4.92 \%)$. On evaluation of the causality of ADRs, majority were found to be Possible (59.02\%). The severity assessment showed that most of the patients ADRs were of moderate level (50.82\%).Some patients required treatment withdrawal and replacement with other drug and most of the patients were managed with supportive medication without removing anti-tubercular drug from their treatment regimen.

Conclusions: ADRs are major factor limiting completion of drug therapy under RNTCP and occurrence of drug resistance which requires attention of all health care professionals.
\end{abstract}

Keywords: Adverse drug reactions, Antitubercular drugs, Causality assessment, Severity assessment

\section{INTRODUCTION}

Multidrug-resistant tuberculosis (MDR-TB) is an increasing global problem, with most cases arising from a mixture of physician error and patient non-compliance during treatment of susceptible TB. The extent and burden of MDR-TB varies significantly from country to country and region to region. It should be stressed that MDR-TB is a man-made phenomenon - poor treatment, poor drugs and poor adherence lead to the development of MDR-TB. Use of inadequate treatment in patients with drug-resistant TB strains will fail to cure a significant proportion of such cases and can create even more resistance to the drugs in use. Ongoing transmission of established drug-resistant 
strains in health care facilities is also believed to be a major source of new drug resistant cases. ${ }^{1}$

Globally in 2016, following WHO guidance issued in May 2016, all cases of rifampicin-resistant TB (RR-TB), including those with multidrug-resistant TB (MDR-TB), should be treated with a second-line MDR-TB treatment regimen. Globally in 2015, there were an estimated 480 000 new cases of MDR-TB and an additional 100000 people with rifampicin-resistant TB who were also newly eligible for MDR-TB treatment; India, China and the Russian Federation accounted for $45 \%$ of these cases. ${ }^{2}$

India is one of the high burden countries for tuberculosis as well as drug-resistant tuberculosis. As per WHO's "Global Tuberculosis Report, 2015", India account for an estimated 480,000 new cases of MDR-TB and an additional 100,000 people with rifampicin resistant TB (RR-TB). People with rifampicin resistant TB are now eligible for the same treatment as people with MDR TB. ${ }^{3}$

Multidrug-resistant tuberculosis (MDR-TB) is defined as resistance to at least isoniazid and rifampicin. ${ }^{4}$

Treatment of MDR-TB is difficult, complicated, much costlier, challenging and needs experience and skills. Reserve drugs are frequently associated with very high rates of unacceptable adverse drug reactions, needing frequent interruption and change of regimen.

Therefore, it is imperative to monitor and treat adverse drug reactions developed by the patients. This approach ensures better compliance of patients and good treatment outcome. At the same time, data regarding ADRs of second-line anti-tubercular drugs in India are scanty. Hence, the aim of this study was to assess the adverse drug reactions of second-line anti-tubercular drugs used to treat MDR-TB in India. 5

\section{Aims and objectives}

- Adverse drug reaction monitoring in patients of MDR-TB at Tertiary Care Hospital.

- Identification of types and frequency of adverse drug reactions in Intensive and continuation phase.

- To evaluate the incidence of treatment discontinuation in relation to ADRs.

- To assess casualty and severity of the reported adverse drug reactions.

\section{METHODS}

Present study was carried out at Drug Resistance Tuberculosis Centre of Govt. Medical College, Aurangabad, Maharashtra, with prior approval of institutional Ethics Committee.

It was a prospective, observational, open label, longitudinal, descriptive clinical study.

\section{Inclusion criteria}

- $\quad$ Patient of either sex of age more than 18 years -45 years with tuberculosis.

- Diagnosed cases of MDR- TB, enrolled under RNTCP program.

- Agreed to adhere tuberculosis treatment regime prescribed.

- Patient who provide written informed consent and ready to give follow up

\section{Exclusion criteria}

- $\quad$ History of Patients receiving ART Treatment

- Patients with deranged Liver and Kidney function tests.

- $\quad$ History of patient suffering from any other chronic disease condition requiring any concomitant medication.

- Patients those were transferred to XDR-TB/whose diagnosis was changed.

- $\quad$ Not ready to give informed consent.

- Not ready to give follow up.

\section{Procedure}

- $\quad$ Patients for this study were included from all patients diagnosed to have MDR-TB (Isoniazid and Rifampicin resistance) and rifampicin resistance by DST and admitted in Drug Resistance Tuberculosis Centre, in Govt. Medical College Aurangabad.

- Data was collected from January 2015 to December 2016.

- All study subjects were evaluated after written informed consent.

- Thorough detailed history was taken regarding the demographic profile, present complaints, past history of tuberculosis, history of any addiction, family history of Tuberculosis.

- Detailed general and systemic examination was done to find out any abnormalities.

- Body mass index (BMI) was calculated in all the patients.

- Pre-treatment investigations done included informed consent, urine for albumin, sugar and pregnancy test for female patients (if 18 to $45 \mathrm{yrs}$. old), complete haemogram, renal and liver function test, Thyroid function test, psychiatric evaluation, Audiometry (SOS), Vision Acuity Test (SOS).

\section{Treatment regimen}

The standardized regimen consisted of an intensive phase (IP) of 6-9 months with 6 drugs, namely kanamycin (Km), ofloxacin (Ofx) (now levofloxacin), ethionamide (Eto), pyrazinamide $(\mathrm{Z})$, ethambutol $(\mathrm{E})$, and cycloserine $(\mathrm{Cs})$ given daily. This was followed by a continuation phase (CP) of 18 months of 4 drugs, namely Ofx (now levofloxacin), Eto, E and Cs. At the end of 6 months of 
treatment, if the fourth month culture remained positive, the IP was extended for a further 3 months. Doses of the drugs were chosen according the weight range to which patient belonged.

All patients enrolled to the study were treated with a daily supervised regimen. All patients were monitored daily for adverse drug reactions after starting regimen till the patients remains admitted in hospital and later followed up personally or telephonically at regular intervals of 2 monthly bases and will be asked questions regarding possible adverse drug reactions of the drug which are prescribed to them. In between the 2 monthly follow up in OPD, telephonic questioning regarding adverse drug reactions will be asked on the any day of first week of every month. Anticipated ADRs will be identified and assessed.

The causality of adverse drug reactions will be assessed as per Naranjo's causality assessment scale, for severity of the adverse drug reactions as per Modified Hartwig-Siegel Scale. At the end of the study, these adverse event records will be analyzed and statistically interpreted.

\section{Parameters were studied}

1. Incidence of adverse drug reactions in anti-TB (AKT) agents.

2. Severity of adverse drug reaction using Modified hartwig-siegel scale.

3. Causality of adverse drug reaction using Naranjo's scale.

\section{RESULTS}

Table 1: Demographic profile of patents.

\begin{tabular}{|c|c|c|c|}
\hline Parameters & & Number & $\begin{array}{l}\text { Percentage } \\
(\%)\end{array}$ \\
\hline \multirow{2}{*}{ Gender } & Male & 71 & 65.74 \\
\hline & Female & 37 & 34.25 \\
\hline \multirow{4}{*}{$\begin{array}{l}\text { Age group } \\
\text { (in years) }\end{array}$} & $<20$ & 13 & 12.03 \\
\hline & $21-30$ & 37 & 34.25 \\
\hline & $31-40$ & 42 & 38.89 \\
\hline & $41-45$ & 14 & 12.96 \\
\hline \multirow{5}{*}{$\begin{array}{l}\text { Weight } \\
\text { bands (Kg) }\end{array}$} & $<16$ & 00 & 00 \\
\hline & $16-25$ & 00 & 00 \\
\hline & $26-45$ & 68 & 62.96 \\
\hline & $46-70$ & 37 & 34.25 \\
\hline & $>70$ & 03 & 2.77 \\
\hline \multirow{3}{*}{ Addictions } & Alcoholic & 20 & 18.51 \\
\hline & smokers & 16 & 14.81 \\
\hline & $\begin{array}{l}\text { Tobacco } \\
\text { chewer }\end{array}$ & 28 & 25.92 \\
\hline
\end{tabular}

A total number of $n=108$ patients who were on DOTS plus therapy were included in this study around $71(65.74 \%)$ were male and $37(34.25 \%)$ were female. Patients of different age group ranging from 18-45 years were reported in the study. 13 patients were under 20 years of age $(12.03 \%)$ followed by 42 resides in the age of $31-40$ (38.89\%), $21-30$ of 37 patients $(34.25 \%)$. Rest were in 4145 years age group $(12.96 \%)$ (Table 1$)$.

Table 2: Incidence of Adverse Drug reactions.

\begin{tabular}{|lll|}
\hline Type of ADR & $\begin{array}{l}\text { No. of } \\
\text { patients }\end{array}$ & $\begin{array}{l}\text { Percentage } \\
(\%)\end{array}$ \\
\hline Gastrointestinal & 32 & 52.45 \\
\hline Nausea, vomiting & 21 & 34.43 \\
\hline Anorexia & 02 & 3.28 \\
\hline Epigastric discomfort & 05 & 4.39 \\
\hline Change of taste & 02 & 3.28 \\
\hline Diarrhea & 02 & 3.28 \\
\hline Hepatitis & 01 & 1.64 \\
\hline Ototoxicity & 07 & 11.48 \\
\hline $\begin{array}{l}\text { Decreased hearing } \\
\text { (B/L SNHL+Tinnitus) }\end{array}$ & 05 & 8.20 \\
\hline Tinnitus +Vertigo & 02 & 1.64 \\
\hline Psychiatric manifestations & & \\
\hline $\begin{array}{l}\text { Insomina+Suicidal } \\
\text { Tendencies }\end{array}$ & 06 & 9.84 \\
\hline Depression & 02 & 3.28 \\
\hline Altered behavior & 01 & 1.64 \\
\hline Insomnia+ Suicidal & 01 & 1.64 \\
\hline Tendencies+Depression & 01 & 1.64 \\
\hline $\begin{array}{l}\text { Hallucination + Suicidal } \\
\text { Tendency }\end{array}$ & 01 & 1.64 \\
\hline Peripheral neuropathy & 02 & 3.28 \\
\hline Vision defect & 02 & 3.28 \\
\hline Impaired visual acuity & 02 & 3.28 \\
\hline Color discrimination & 00 & 00 \\
\hline Skin & 03 & 4.92 \\
\hline Pruritus with rash & 02 & 3.28 \\
\hline Pruritus without rash & 01 & 1.64 \\
\hline $\begin{array}{l}\text { Injection site pain and } \\
\text { swelling }\end{array}$ & 04 & 6.55 \\
\hline Renal dysfunction & 02 & 3.28 \\
\hline Deranged RFT & 02 & 3.28 \\
\hline Musculoskeletal & 03 & 4.92 \\
\hline Arthralgia & 61 & 4.92 \\
\hline Total & 100 \\
\hline & & \\
\hline
\end{tabular}

Out of these 108 patients 48 patients developed 61 ADRs of various types (Table 2). Among the 61 reported ADRs, most were observed in males $(30 / 62.50 \%)$ and remaining $(18 / 37.50 \%)$ were females. The overall incidence of ADRs was $56.48 \%$. ADRs in this study were categorized according to the systems affected like gastrointestinal system, ototoxicity, psychiatric manifestations and other systems like skin, vestibular, musculoskeletal etc. Majority of ADRs were related to gastrointestinal system (32 cases $/ 52.45 \%$ ) followed by ototoxicity (7cases/11.48\%), Psychiatric Manifestations (06 Cases/9.84\%), other systems (16 cases). Nausea and 
Vomiting was the most common ADR (21/34.43\%) followed by Ototoxicity (6/9.84\%), Inj. site pain swelling $(4 / 6.55 \%)$.

Out of the 108 drug resistance tuberculosis patients, patients were divided in the different weight bands according to it $68(62.96 \%)$ patients in weight band 26 $45 \mathrm{~kg}$ followed by 37 (34.25\%) patients in weight band 46$70 \mathrm{~kg}$. Drug resistance pattern in out of 108 patients, 57 $(52.78 \%)$ patients showing rifampicin mono-resistance while $51(47.22 \%)$ patients showing Isoniazid and Rifampicin Resistance.

The main action taken in patients with detected ADR was Withhold and Replacement of drug seen. The action mainly was taken when patients with psychiatric ADRs required withdrawal of cycloserine which was replaced with PAS.one patients required pyrazinamide withdrawal for peripheral neuropathy. While Kanamycin was replaced with PAS in patients suffers from ototoxicity (Table 3 ).

Table 3: Causality of ADRs induced by anti TB drugs according to Naranjo algorithm.

\begin{tabular}{|llll|}
\hline Sr. no & Type & No. of patients & Percentage \\
\hline 1 & Probable & 25 & 40.98 \\
\hline 2 & Possible & 36 & 59.02 \\
\hline 3 & Certain & 00 & 00 \\
\hline Total & & 61 & 100 \\
\hline
\end{tabular}

The causality assessment of ADRs revealed that 13 $(21.31 \%)$ cases were detected as Definite, 19 (31.14\%) as possible and $29(47.54 \%)$ as probable reactions. The Severity assessment of ADRs revealed that 31 (50.82\%) cases were mild, $24(39.34 \%)$ were Moderate and 06 (9.84\%) sever ADR observed (Table 4).

Table 4: Severity of ADRs induced by anti TB drugs according to Modified Hartwig-sigel Scale.

\begin{tabular}{|lll|l|}
\hline Sr. no. & Type & No. of patients & Percentage \\
\hline 1 & Mild & 25 & 40.98 \\
\hline 2 & Moderate & 31 & 50.82 \\
\hline 3 & Severe & 05 & 8.91 \\
\hline Total & & 61 & 100 \\
\hline
\end{tabular}

\section{DISCUSSION}

The present observational study has evaluated a DOTSPlus programme, with special reference to Adverse Drug effects in which standard treatment of drug resistant tuberculosis cases as per RNTCP guidelines has been started in this DR-TB Centre.

In the present study of 108 patients, the age group ranged from 18 to 45 years. Maximum number of cases were in the age group $31-40 y r s$ (38.89\%) followed by $21-30 y r s$ (34. 25\%). The median age of the patients in present study was 31.78 years, as compared to the reports in which the median age was 28 years. $^{6}$ And in another study it was reported as 26 years respectively. ${ }^{7}$

In the present study, majority of the patients were males71 $(65.74 \%)$ and Females 37 (34.25\%). In the present study, majority of the patients were males $71(65.74 \%)$ and Females 37 (34.25\%). similar observations were noted by authors in a study (males $65.79 \%$ and females $34.21 \%$ ). ${ }^{2}$ and proportion of males to females was $54.54 \%$ and $45.46 \%$ respectively. ${ }^{6}$

Weight band: Of the 108 drug resistance tuberculosis patients in this study, majority of patients were in the weight band of 26 to $45 \mathrm{Kg}(62.96 \%)$. Whereas, a study observed that majority patients were above $4 \mathrm{Kg}(51.43 \%)$. Majority of the drug resistance tuberculosis patients were underweight before the start of treatment.

In Present study, rifampicin mono resistance was found in $52.78 \%$, while both isoniazid and rifampicin resistance were found in $47.22 \%$ patients. Initially, when our DR-TB center started only solid cultures were available in the program due to which both rifampicin and isoniazid resistance was reported together. As line probe assay became available, rifampicin mono resistance cases started getting picked up.

In this study ADRs were observed in $56.48 \%$ patient's, a finding comparable to present study reports notified in different studies. ${ }^{7-9}$

The ADR reported in present study were, Gastrointestinal, Ototoxicity, Psychiatric manifestations, Injection site swelling/pain, Arthralgia, Skin, Renal Involvement, Vision defect, peripheral neuropathy.

Gastro intestinal symptoms were most common adverse reaction observed in this study that is $32(52.45 \%)$ similar to other studies. ${ }^{9-11}$ on the contrary other studies have found observed gastrointestinal ADRs in 42\%, 60\% and $100 \%$ patients respectively. ${ }^{7,8,12,13}$ Hepatotoxicity was noted in $1(1.64 \%)$ patient only. Similarly finding were reported other authors. ${ }^{11,13}$

They were mild but required immediate treatment. These gastrointestinal symptoms occurred mostly within a week of starting treatment. No patient required alteration in DOTS-Plus treatment due to gastrointestinal ADRs.

Ototoxicity 7 (11.68\%) was second most common ADR observed in this study of which decreased hearing 5 and tinnitus and vertigo in 2 patients These findings were similar to observations in a study which reported ototoxicity as second most common ADR after gastrointestinal ADR and frequency of ototoxicity. ${ }^{2,11,14}$ whereas another study reported ototoxicity in $15 \%$ patients. ${ }^{12}$ Kanamycin was withdrawn in $80 \%$ of these patients and substituted with PAS ( $\mathrm{p}$ - amino salicylic acid). 
Psychiatric $06(9.84 \%)$ manifestations were the third most common adverse reaction in this study of which insomnia was the most common followed by suicidal tendency, depression and altered behavior in descending order. Psychiatric ADRs were less common in this study as compared to $15.9 \% .^{7}$ and $15 \% .{ }^{15}$ in other studies. All patients with psychiatric manifestation required withdrawal of cycloserine which was replaced with PAS (P-amino salicylic acid).

Injection site swelling/pain 4 (6.55\%) was fourth common ADR observed in this study. In contrast, it was reported in a study that injection site swelling/pain seen in $21.05 \%$ patients. ${ }^{8}$ None of the patients required withdrawal of injection Kanamycin.

Arthralgia 3 (4.92\%) was fifth common ADR observed in this study. Similar observation was seen in $4.5 \%$ and $7.94 \%$ respectively. ${ }^{9,11}$ In contrast, it was observed in the studies that arthralgia was seen in $31 \%$ and $23.68 \%$ patients. ${ }^{8,14}$

Skin Adverse drug reactions ADR observed in this study was $3(4.92 \%)$ of which pruritus without rash in 1 and pruritus with rash in 2 patient. Frequency of skin reaction found in this study is similar $4 \%, 1.58 \%$ and $4.5 \% .^{7,11,16} \mathrm{On}$ the one of the study reported cutaneous reactions in $43.3 \%$ patients. $^{13}$

Renal involvement was seen $2(3.28 \%)$ patients in this study which is similar to observation noted in different other studies $1.58 \%, 2.7 \%$ and $2 \%$ respectively. ${ }^{9,11,12}$ Renal involvements were seen in the form of borderline derangement of serum creatinine $(2 \mathrm{mg} \%)$ which improved in few weeks and none required withdrawal of injection kanamycin.

Other ADR including Visual defect in 2 (3.28\%), Peripheral Neuropathy 2(3.28). similar findings seen in a study with frequency of visual disturbance $1(0.9 \%)$ and peripheral neuropathy $3(2.7 \%){ }^{9}$

In present study Causality assessment of 61 ADRs was done by Naranjo's Causality Scale, out of 61 ADRs,36(59.02\%)into possible category, And $25(40.98 \%)$ fall into Probable category. None of categorized into 'Certain' category.

The severity of ADRs in the present study was assessed by Modified Hartwig and sigel scale. The distribution of 61 ADRs as Mild 40.98\%, moderate $50.82 \%$ and sever $8.91 \%$, as the study population the patients was hospitalized for ADRs, higher number of ADRs belonged to "Moderate "grade.

\section{CONCLUSION}

Drugs for treating MDR-TB strains involve a long-term exposure and have greater toxicity effects. A high frequency of adverse drug reactions is one of the major challenge in the treatment of MDR-TB Pharmacovigilance now become important component of drug treatment, Drug therapy and active Pharmacovigilance goes hand in hand.

The present study evaluated pattern and frequency of adverse drug reactions in patients receiving treatment for Multi-drug resistant tuberculosis and assessed their severity and causality. A majority of ADRs is possible in causality assessment and more of them are seems to be treatable and preventable.

\section{ACKNOWLEDGEMENTS}

Authors would like to thank the department of ENT and Ophthalmology, Govt. Medical College, Aurangabad (MS) INDIA for their kind help in analysis of ADRs. Authors would also like to acknowledge the generous support from all concerned in the Chest TB department of GMC, Aurangabad, India.

Funding: No funding sources Conflict of interest: None declared

Ethical approval: The study was approved by the Institutional Ethics Committee

\section{REFERENCES}

1. Directorate General of Health Services, Ministry of Health and Family Welfare, Government of India; New Delhi. Central TB Division. Guidelines on Programmatic Management of Drug Resistant TB (PMDT) in India. New Delhi: May 2012.

2. WHO. Global Tuberculosis Report 2016. [Internet]. 2016. Available at: http://scholar.google.com/scholar?hl=enandbtnG=Sea rchandq=intitle:No+Title\#0

3. National and state statistics TB statistics for India. Organisation WH, Control GT. MDRTB Statistics for India. 2015:1-9.

4. Joseph P, Desai VB, Mohan NS, Fredrick JS, Ramachandran R, Raman B, et al. Outcome of standardized treatment for patients with MDR-TB from Tamil Nadu, India. The Ind J of Med Resea. 2011 May;133(5):529.

5. Prasad R. Management of multi-drug resistant tuberculosis Practitioner's view point. Indian Journal of Tuberculosis. 2007;54:3-11.

6. Arora VK, Sarin R, Singla R, Khalid UK, Mathuria K, Singla N, et al. DOTS-Plus for patients with multidrug-resistant tuberculosis in India: early results after three years. Ind $\mathbf{J}$ of Che Disea and All Scienc. 2007;49(2):75.

7. Singla R, Sarin R, Khalid UK, Mathuria K, Singla N, Jaiswal A, et al. Seven-year DOTS-Plus pilot experience in India: Results, constraints and issues. The International Journal of Tuberculosis and Lung Disease. 2009;13:976-81.

8. Hire R, Kale AS, Dakhale GN, Gaikwad N. A prospective, observational study of adverse reactions to drug regimen for multi-drug resistant pulmonary 
tuberculosis in Central India. Medit J of Hem and Infec Disea. 2014;6(1).

9. Singh BD, Ghulam H, Kadri SM, Qureshi W, Kamili MA, Singh $H$ et al. Multidrug-Resistant and extensively drug resistant tuberculosis in Kashmir, India. J Infect Dev Ctries. 2010;4(1):19-23.

10. Kapadia VK, Tripathi SB. Analysis of 63 patients of MDR TB on DOTS plus regimen: An LG hospital, TB Unit, Ahmadabad experience. Guj Med J. 2013;68(2);52-7.

11. Singh R, Gothi D, Joshi JM. Multidrug Resistant Tuberculosis: Role of Previous Treatment with Second Line Therapy on Treatment Outcome. Lung India. 2007;24:54-7.

12. Furin JJ, Mitnick CD, Shin SS, Bayona J, Becerra MC, Singler JM, et al. Occurrence of serious adverse effects in patients receiving community-based therapy for multidrug-resistant tuberculosis. Int $\mathrm{J}$ tuberc lung dis. 2001;5(7):648-55

13. Jain K, Desai M, Dikshit RK. Treatment outcome of standardized regimen in patients with multidrug resistant tuberculosis. J Pharmacol Pharmacother. 2014;5(2):145-9.
14. Bloss E, Kukša L, Holtz TH, Riekstina V, Skripčonoka V, Kammerer S, et al. Adverse events related to multidrug-resistant tuberculosis treatment, Latvia, 2000-2004. Int J tuberc lung dis. 2010 Mar $1 ; 14(3): 275-81$.

15. Törün T, Güngör G, Özmen I, Bölükbaşı Y, Maden E, Bıçakçı B, Atac G, Sevim T, Tahaoğlu K. Side effects associated with the treatment of multidrug-resistant tuberculosis. Int $\mathbf{J}$ tuberc lung dis. 2005 Dec 1;9(12):1373-7.

16. Wai yew W, Kuen CC, Hung CC, Cheeuk MTC. Outcomes of patients with multidrug resistant pulmonary tuberculosis treated with Ofloxacin/Levofloxacin containing regimens. Chest. 2000;177:744-51.

Cite this article as: Baig MS, Kale MR, Lamb A. A prospective observational pharmacovigilance study of adverse drug reaction monitoring in patients of MDR - TB at tertiary care hospital. Int J Basic Clin Pharmacol 2018;7:1291-6. 